\title{
Role of HPV E6 proteins in preventing UVB-induced release of pro-apoptotic factors from the mitochondria
}

\author{
Sabrina Leverrier • Daniele Bergamaschi - Lucy Ghali - Ayodele Ola • Gary Warnes • \\ Baki Akgül · Ken Blight • Ramón García-Escudero • Aubin Penna • Ayad Eddaoudi • \\ Alan Storey
}

Published online: 31 December 2006

(C) Springer Science + Business Media, LLC 2006

\begin{abstract}
Apoptotic elimination of UV-damaged cells from the epidermis is an important step in preventing both the emergence and expansion of cells with carcinogenic potential. A pivotal event in apoptosis is the release of apoptogenic factors from the mitochondria, although the mechanisms by which the different proteins are released are not fully understood. Here we demonstrate that UV radiation induced the mitochondrial to nuclear translocation of apoptosis inducing factor (AIF) in normal skin. The human papillomavirus (HPV) E6 protein prevented release of AIF and other apoptotic factors such as cytochrome $c$ and Omi from mitochondria of UV-damaged primary epidermal keratinocytes and preserved mitochondrial integrity. shRNA silencing of Bak, a target for E6-mediated proteolysis, demonstrated the requirement of Bak for UV-induced AIF release and mitochondrial fragmentation. Furthermore, screening non-melanoma skin cancer biopsies revealed an inverse correlation between
\end{abstract}

S. Leverrier · D. Bergamaschi · L. Ghali · A. Ola · B. Akgül ·

R. García-Escudero · A. Storey $(\square)$

CR-UK, Skin Tumour Laboratory, Centre for Cutaneous

Research, 4 Newark Street,

E1 2AT London, UK

e-mail: alan.storey@cancer.org.uk

G. Warnes · A. Eddaoudi

CR-UK FACS Lab, CRUK LRI,

44, Lincoln's In Fields,

WC2A 3PX London, UK

K. Blight

CR-UK EM Unit, LRI,

44, Lincoln's In Fields,

WC2A 3PX London, UK

A. Penna

Institut de Genomique Fonctionnelle,

141 rue de la Cardonille 34396, Montpellier, France
HPV status and AIF nuclear translocation. Our results indicate that the E6 activity towards Bak is a key factor that promotes survival of HPV-infected cells that facilitates tumor development.

Keywords AIF $\cdot$ Bak $\cdot H P V \cdot$ Skin cancer $\cdot$ UV

\section{Introduction}

Apoptosis, or programmed cell death, triggers a series of events that lead to the efficient elimination of supernumerary cells during development or the selective killing of badly damaged and potentially tumorigenic cells. Of the two main pathways of apoptosis that can be triggered, either through cell surface death receptors or an intrinsic mechanism involving mitochondria, the latter is most often seen in vertebrate cells in response to a variety of genotoxic insults [1].

Present address:

L. Ghali

Department of Biomedical Sciences, School of Health and Social

Sciences,

Middlesex, EN3 4SA, UK

R. García-Escudero

Cellular and Molecular Biology, CIEMAT,

Av Complutense 22 28040, Madrid, Spain

S. Leverrier

Department of Molecular Biology and Biochemistry, Center for

Immunology,

3240 McGaugh Hall, UC Irvine, Irvine, CA 92697, USA

G. Warnes

Institute of Cell and Molecular Science,

4 Newark Street, London, E1 2AT, UK 
A pivotal event in this process is the loss of mitochondrial outer membrane potential (MOMP), a complex event which is thought to depend on two key pro-apoptotic members of the Bcl-2 family, Bax and Bak, and is considered as critical in commitment to cell death [2-4]. When activated, Bax and Bak can form multimeric complexes containing lipids that are able to form supra-molecular openings in the mitochondrial outer membrane leading to loss of MOMP and the selective release of proteins located in the inter-membrane mitochondrial space (IMS) [5-7]. Proteins released from the IMS can promote apoptosis via two general mechanisms either involving cytosolic events leading to caspase activation, or a second separate pathway that depends on nuclease activities [8]. However, both the composition, structure and biochemical characteristics of the mitochondrial intramembranous complexes formed by Bax and Bak, as well as their ability to differentially regulate the release of IMS proteins, remain unclear. The release of IMS proteins such as cytochrome $c$, Smac/DIABLO and Omi/HtrA2 results in caspase activation, whereas release of other factors such as AIF and Endonuclease G leads to their translocation to the nucleus where they participate in DNA condensation and large scale cleavage that is distinct from oligonucleosomal fragmentation [8-10].

While the release of AIF from the mitochondria is now recognized as being an important step in apoptotic signaling, there are differing reports as to the nature of the molecular determinants governing AIF release in response to diverse apoptotic stimuli in different cell types [11]. Although there are reports indicating AIF release is dependent upon upstream caspase activation and cytochrome $c$ release, they are counterbalanced by evidence that indicates AIF release occurs independently of caspase activity $[12,13]$. Experiments probing how mitochondrial membrane permeability is achieved in molecular terms have also yielded different results with regard to cytochrome $c$ and AIF release. In in vitro reconstitution experiments using Bcl-2 family members, channels with distinct molecular cut offs were generated that allowed the passage of small molecules such as cytochrome $c$ but not of the larger AIF [14]. In contrast to this, other reports suggest that composite channels consisting of t-Bid, Bax and mitochondrial lipids formed giant pores capable of releasing molecules up to $2 \mathrm{MDa}$ [15].

Solar UV radiation damages DNA, is highly mutagenic and is recognized as an important environmental human carcinogen. UV is the main aetiological agent that results in over one million cases of non-melanoma skin cancer (NMSC) in the USA and $>60,000$ cases in the UK annually [16, 17]. UV is a powerful apoptotic inducer in human epidermis leading to the production of "sunburn" cells that display hallmarks of apoptosis including nuclear chromatin condensation and fragmentation [18]. The failure to repair damaged DNA or to remove severely damaged cells by apoptosis can lead to the propagation of deleterious somatic mutations and ultimately to carcinogenesis [19]. UVB has been shown to be a physiologically important inducer of Bak activity that results in activation and stabilization of the protein in the epidermis with a concomitant induction of apoptosis, an activity that is independent of the p53 tumor suppressor [20]. In addition to $\mathrm{UVB}$, there is an emerging role for cutaneous human papillomaviruses (HPV) in NMSC development with most tumors, including those that contain HPV, occurring at body sights exposed to solar radiation [21-23]. Specific patient groups, such as iatrogenically immunosuppressed organ transplant patients, have a well-documented 50-100 fold increased risk of developing NMSC and these lesions also show a high carriage rate of HPV [22, 24]. The HPV E6 protein effectively inhibits UV-induced apoptosis, an activity that is mediated at least in part by the abrogation of Bak activity through proteolytic degradation [20, 25]. HPV-positive NMSC display low apoptotic rates and have lower levels of Bak protein than HPV-negative NMSC. The suppression of UV-induced apoptosis has been proposed as a general mechanism by which cutaneous HPVs can promote skin cancer development [26, 27].

We have further investigated the anti-apoptotic effects of the HPV E6 protein with regard to the release of proapoptotic factors from the mitochondria, both in monolayer and organotypic cell cultures and in skin cancer biopsy material. The findings both shed new light on the mechanisms involved in UV-induced release of apoptogenic factors from mitochondria and reveal important physiological implications for skin cancer development.

\section{Results}

HPV E6 inhibits UV-induced mitochondrial morphological changes and the release of pro-apoptotic factors

Having previously demonstrated that HPV E6 interferes with UVB-induced apoptosis in a number of different cell types, we were interested to investigate the consequences on the mitochondrial apoptotic pathway [20, 28]. HT1080 cells that express UV-inducible Bak and p53 were transfected with the HPV E6 gene of HPV types 5, a $\beta$-papillomavirus found in NMSC, and HPV 18, a mucosal HPV found in anogenital cancers [29]. We first compared HPV expressing and control cells for morphological changes induced by UVB by electron microscopy (Fig. 1(A)). Mitochondria of control cells were found to undergo fragmentation following UVB treatment and close inspection revealed pinch points that are formed during the fragmentation process and are believed to be sites where Bak multimers coalesce [30]. In contrast, in cells expressing either of the E6 proteins mitochondrial con- 
densation and fragmentation are significantly reduced and no pinch points were observed.

To investigate how E6 was able to prevent the mitochondrial fragmentation process we examined the expression levels of key apoptotic regulators such as Bak, p53 and AIF after UVB exposure (Fig. 1(B)). In agreement with our previous reports, following UV irradiation the HPV18 E6 protein targeted both Bak and p53 for proteolysis, while the HPV5 E6 protein only promoted Bak degradation [20]. UV treatment did not affect AIF levels and this was not influenced by expression of either E6 protein, indicating that AIF is not a target for E6-mediated proteolysis. As AIF is known to exert its apoptotic activity at the nuclear level, we then analyzed whether AIF translocation was affected by UV treatment and whether E6 modulated this translocation. Immunocytochemical analysis of AIF sub-cellular localization showed that UVB caused AIF to translocate from the mitochondria and accumulate in the nucleus in control cells (Fig. 1(C)). In E6-expressing cells however AIF was located in the mitochondria before UV treatment and did not undergo translocation to the nucleus. To further confirm these observations on the cell cultures as a whole and to obtain quantitative data on AIF translocation, we developed a FACS technique capable of quantifying the level of protein expression at different sub-cellular locations (Fig. 1(D)). These fractionation experiments revealed that translocation of AIF was inhibited in the E6 cells and also that the release of other IMS proteins such as cytochrome $c$ and Omi was also prevented (Fig. 1(E)). FACS profiles of sub-cellular fractions showed a decrease in mitochondrial levels of AIF with a concomitant increase in the nuclear compartment following UV treatment. In addition, lower levels of both cytochrome $c$ and Omi were present in the mitochondrial fraction after UV treatment. This new method of FACS analysis also permits quantification of the data, this is shown in the bar graphs. Together, these results demonstrate that E6 expression causes a major dysfunction in mitochondrial apoptosis by preventing both morphological and other biochemical changes. The degradation of $\mathrm{p} 53$ by HPV18 E6 but not by HPV5 E6 indicates that the inhibitory effects of E6 appear to be independent of p53 status.

Bak is required for AIF release from the mitochondria after UV irradiation

Although the pro-apoptotic role of Bak has been mostly studied in association with its close homologue Bax, it has been clearly established that Bax and Bak can mediate apoptosis independently [5, 31]. The ability of Bax and Bak to interact with various partners to regulate specifically the release of pro-apoptotic factors from the mitochondria also seems to differ [32]. However, as Bax is not a transcriptional target of p53 in human epidermal cells following DNA damage [33], we focused further experiments on Bak.
To determine the importance of Bak degradation mediated by E6 and whether this was directly involved in the failure of AIF to translocate to the nucleus after UV, we established monoclonal HT1080 cell lines in which the expression of Bak was silenced by specific shRNAs. The specificity of Bak silencing was established using western blotting to Bak and a control molecule, EGFP (Fig. 2(A)), and both the BI and BII lines yielded similar rersults. Bak-silenced cells displayed a significantly lower apoptotic rate (assessed by Annexin Vbinding) following UVB treatment, that was accompanied by a lower decrease in mitochondrial membrane potential, $\Delta \Psi m$, when compared to control cells (Fig. 2(B) and (C)). The effects on both apoptotic rate and mitochondrial membrane potential were most dramatic at early time points after UV treatment. This suggests that Bak plays a key role by participating in an early wave of apoptosis, and that at later time points other Bak-independent mechanisms then play an increasingly important role in apoptosis in this system.

Morphological changes in the mitochondria were assessed using EM as described above, but no fragmentation or pinch points were detected in UVB-irradiated Bak silenced cells and additionally, no translocation of AIF was observed (Fig. 2(D) and (E)). The partial resistance of Bak-silenced cells to apoptotic stimuli is consistent with data obtained from $\mathrm{Bak}^{-/-}$MEFs following either UVC or staurosporine treatment $[5,31]$. In addition to the observed effects of E6 on AIF sub-cellular localisation as determined by confocal microscopy and FACS, we have performed conventional cell fractionation experiments on E6-expressing and Bak silenced cells to confirm further our findings. AIF mitochondrial to nuclear translocation was effectively inhibited in both the HT1080 HPV5 E6-expressing cells and in the HT1080 Bak silenced cell line derivative BII where AIF was retained in the mitochondrial cell fraction after UV exposure (Fig. 2(F)). These results demonstrate the requirement of Bak for UVB-induced apoptosis not only at the morphological level but also for the release of different pro-apoptotic IMS proteins. The comparable maintenance of mitochondrial morphology and retention of IMS proteins in E6 expressing cells compared to Bak-silenced cells following UVB exposure suggests that similar mechanisms were operating in each case that preserved mitochondrial integrity.

E6 prevents UV-induced AIF nuclear translocation in regenerated epithelium

The findings that E6 was able to inhibit AIF translocation and release of other IMS proteins suggest that E6 expression is likely to have significant effects in modulating the response of skin to UV. To investigate more closely the physiological relevance of the observations on monolayer cell cultures, we analyzed AIF sub-cellular localization in explant cultures of skin maintained in organ culture, and also 

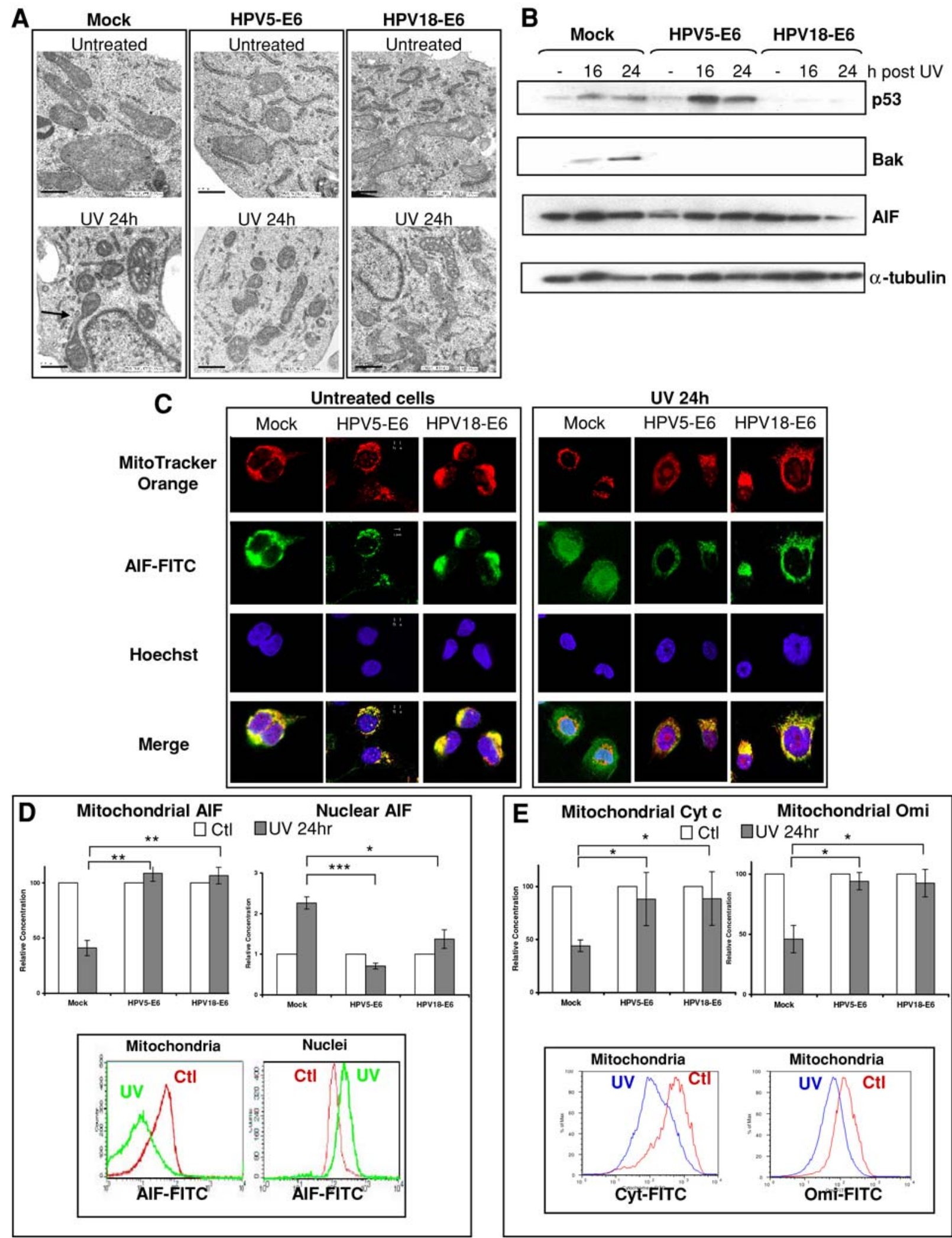
4 Fig. 1 Mitochondrial apoptotic pathway induced by UV irradiation is disrupted in E6 expressing cells. UVB response of stable HT1080 cell lines transfected with empty vector (Mock), HPV5-E6 or HPV18-E6 protein was assessed. (A) E6 protein interferes with UV-induced mitochondrial fragmentation. Mitochondrial morphology of untreated cells or $24 \mathrm{~h}$ after UVB irradiation $\left(15 \mathrm{~mJ} / \mathrm{cm}^{2}\right)$ was visualized by electron microscopy. The UV-induced formation of pinch point (indicated by arrows) was prevented in E6 expressing cells. Images shown are representative of 5 analyzed sections. Bars represent $0.5 \mu \mathrm{m}$. (B) Level of apoptotic factors in E6 cells following UV irradiation. Whole cell lysates prepared from untreated cells, $16 \mathrm{~h}$ or $24 \mathrm{~h}$ after exposure to UVB $\left(15 \mathrm{~mJ} / \mathrm{cm}^{2}\right)$ were resolved by SDS-PAGE and the resulting blot probed with the indicated monoclonal antibodies. p53 accumulation after UV was prevented in HPV18-E6 cells and Bak was degraded in both HPV5E6 and HPV18-E6 cells. The $\alpha$-tubulin level was used as a control to normalize protein loading. (C) Abrogation of UV-induced AIF nuclear relocalization by E6 expression. Three-color confocal microscopy of Mock, HPV5-E6 or 18-E6 cells was assayed before treatment (left

in organotypic keratinocyte cultures of regenerated stratified epithelium that express the HPV E6 protein that can be used to mimic early skin lesions. UVB irradiation of normal skin demonstrated that AIF translocation occurred progressively in skin in vivo, with AIF first being detected in the cytoplasm and then at later time points in the nucleus of the keratinocytes (Fig. 3(A)), suggesting that caspase-independent pathways involving AIF operate in this tissue. Notably, AIF expression was not detected in unirradiated skin explants. To determine whether this was due to lack of expression or possible masking of the antibody epitope in the tissue through complex formation with binding partners such as HSP70, we performed western blotting on whole cell extracts of skin using the same anti-AIF antibody as used in the immunohistochemical analysis (Fig. 3(B)). This confirmed that AIF was expressed in normal skin and, in agreement with our previous observations in cell cultures, that AIF protein levels were unaffected by UV irradiation.

To investigate whether the E6 protein was also able to inhibit AIF translocation in epithelium, primary human epidermal keratinocytes were infected with a recombinant retrovirus expressing the E6 gene of HPV type 8, a virus with very close homology to HPV5, or retrovirus containing empty vector. Reconstituted epidermal sheets were generated by seeding the transduced keratinocytes onto de-epidermalized human dermis and allowing the cells to differentiate over a 14 day period. The reconstituted skin was then irradiated as before for normal skin and harvested $24 \mathrm{~h}$ later (Fig. 3(C)). As noted for untreated normal skin, AIF was not detected in the absence of UVB treatment, in either E6-transduced organotypic cultures or controls. However, $24 \mathrm{~h}$ after UV treatment of the regenerated epithelium AIF was clearly detected in the nuclei of cells transduced with empty retrovirus. By comparison, AIF was detected in epithelium regenerated using E6-transduced keratinocytes but its expression was limited to the cytoplasm and no nuclear staining was observed. This suggests that while UVB may have panel) or $24 \mathrm{~h}$ after UVB irradiation (right panel). The mitochondria staining in red (MitoTracker Orange CMTMRos), AIF (AIF-FITC) in green and nuclei were stained with Hoechst in blue. The pictures shown are representative of three independent immunostaining experiments. Bars represent $5 \mu \mathrm{m}$. (D) AIF and (E) cytochrome $\mathrm{c}$ and Omi release respectively from the mitochondria (left) and relocalization to the nuclei (right) was also assayed by flow cytometric analysis of untreated $(\mathrm{Ctl})$ or UVB-irradiated cells stained with probes and antibodies as in C. Representative plots of AIF-FITC, cyto c-FITC and Omi-FITC signals in mitochondrial and nuclear fractions of HT1080 cells before and after UV treatment are shown. Fluorescence was quantified using a LSR flow cytometer. The bar charts represent means \pm SD from three independent experiments $\left({ }^{* * *} p=0.0001,{ }^{* *} 0.001 \leq p \leq 0.009\right.$, ${ }^{*} 0.01 \leq p \leq 0.09$ calculated with the Student's $t$-Test). (E) Likewise, Cytochrome $c$ (Cyt, left) and Omi (right) release from the mitochondria $24 \mathrm{~h}$ after UVB irradiation was monitored by flow cytometry using selective antibodies. Data shown are mean \pm SD from three independent experiments

initiated early events that lead to activation of AIF, E6 nevertheless prevented the release of the protein from the mitochondria.

\section{AIF expression in non-melanoma skin cancers}

An important role for the AIF-mediated apoptosis pathway has recently been proposed in tumor tissue as well as cultured cells where tumor cells exhibited non-classical features of apoptosis, showing no caspase activation and AIF nuclear localization $[12,34,35]$. Moreover, it has been further suggested that AIF may determine the chemoresistance of non-small-cell lung carcinomas to conventional anti-cancer therapies [36]. In addition, the resistance to loss of MOMP has been defined as a prominent hallmark of cancer cells, although the molecular mechanisms that underlie this defect remains unclear at present [37]. Since squamous cell carcinomas (SCC) that contain HPV DNA sequences have been shown to have a lower apoptotic rates than HPV-negative NMSC [25], we extended our studies to investigate whether AIF played a part in the apoptotic response in skin cancers. To test this idea, we then investigated AIF sub-cellular localization in a series of $17 \mathrm{HPV}$-positive and -negative SCC biopsies. After HPV typing, SCC biopsies that were either positive or negative were selected for analysis.

This immunohistochemical investigation showed that in the HPV-negative tumors, AIF was readily detected and was found to be located in both the cytoplasm and nuclear compartments (Fig. 4). In marked contrast, the HPV-positive SCCs displayed only weak AIF staining that was confined to the cytoplasm. Of the HPV-negative tumors, 8/9 showed strong AIF staining and AIF was detected in the nucleus in 6/9 biopsies, while in the HPV-positive SCCs, only 5/8 lesions showed weak cytoplasmic AIF staining and $0 / 8$ showed any nuclear AIF. Thus, we observe a clear difference in the nuclear localization of AIF, where the protein would be able to exert its apoptotic activity, between HPV-positive and - 

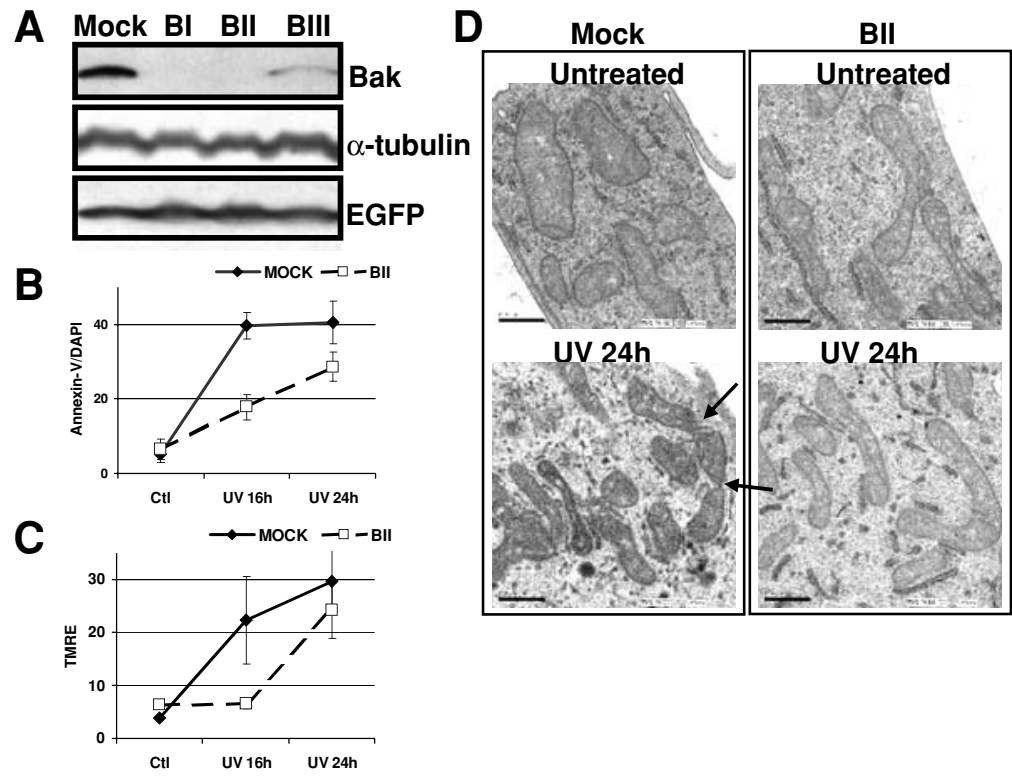

E
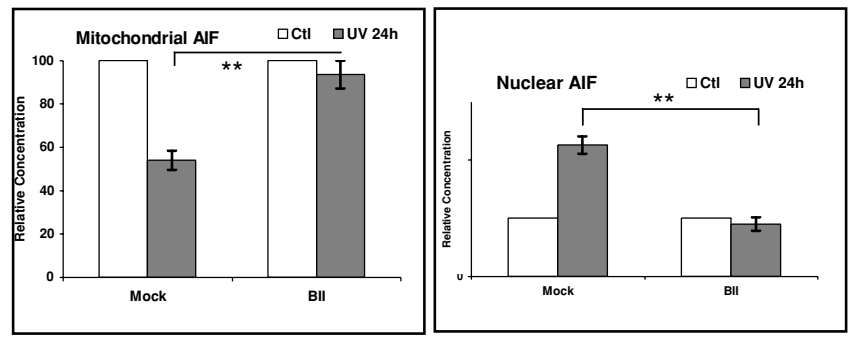

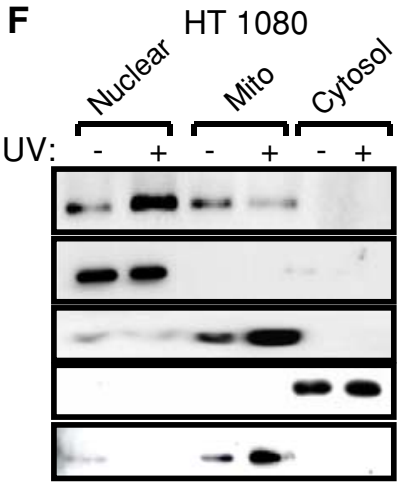

Fig. 2 Bak silencing alters key features of the mitochondrial apoptotic pathway. (A) Silencing of Bak in HT1080 cell line using specific shRNA. Immunoblot analysis of lyzates prepared from monoclonal cell lines expressing Bak shRNA templates (BI, BII and BIII) or the empty vector (Mock) indicated the level of Bak protein. EGFP level was assessed to attest an equal expression of the vector and $\alpha$-tubulin level was used as a control to normalize protein loading. (B) The Baksilenced cell line BII displayed a lower apoptotic rate following UV irradiation. Apoptosis was assessed by flow cytometric detection of Annexin-V/DAPI in control (Mock) and Bak-silenced cells (BII) untreated, $16 \mathrm{~h}$ or $24 \mathrm{~h}$ after UVB exposure $\left(15 \mathrm{~mJ} / \mathrm{cm}^{2}\right)$. The data shown are means \pm SD of three independent experiments. (C) Bak silencing also affected the loss of mitochondrial membrane potential $(\Delta \Psi \mathrm{m})$ induced by UV. Mitochondrial membrane potential was assayed by flow cytometry using TMRE probe. The data presented as the means of three independent experiments \pm SD. (D) Bak participates to the mitochon- drial fragmentation process. Morphological changes before and $24 \mathrm{~h}$ after UVB irradiation of Mock or Bak-silenced cells were evidenced by electron microscopy. The arrows point to the mitochondrial pinch points. Images shown are representative of 5 analyzed sections. Bar represents $0.5 \mu \mathrm{m}$. (E) Bak is required for AIF relocation. Measurement of AIF release from the mitochondria (left panel) and translocation to the nucleus (right panel) was assayed by flow cytometric analysis of Mock and Bak-silenced cells before and $24 \mathrm{~h}$ after UVB irradiation. Data shown are mean \pm SD from three independent experiments ${ }^{* *} 0.001 \leq p \leq 0.009$, calculated with the Student's $t$-Test). (F) Cell fractionation. Nuclear, cytosolic and mitochondrial fractions were isolated from HT1080, HT1080 HPV5E6 or Bak-silenced HT1080 cells (BII) and western blotted for AIF. Markers of the different cellular fractions were as follows; nucleus: PCNA, mitochondria: Cox4, cytosol: actin 


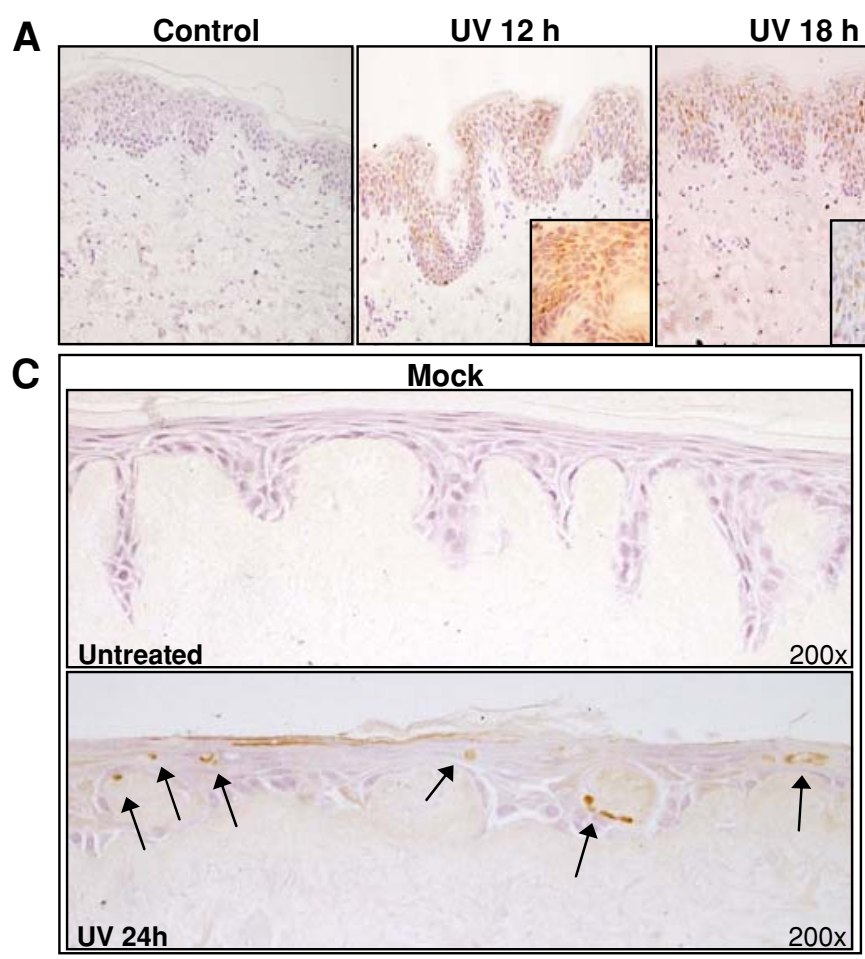

Fig. 3 E6 inhibits UV-induced AIF relocation to the nucleus in normal human skin. (A) UV induces AIF nuclear translocation in human skin. Immunostaining was performed on skin sections at various times $(12 \mathrm{~h}, 18 \mathrm{~h}$ and $24 \mathrm{~h})$ after exposure to UVB $\left(45 \mathrm{~mJ} / \mathrm{cm}^{2}\right)$ using AIF monoclonal antibody (stained brown) and Hematoxylin and eosin counterstain. Pictures were taken at a magnification of $200 \times$ and $1000 \times$ (insets) using conventional microscopy. (B) AIF expression remains stable in human skin. Lysates prepared from primary human keratinocytes untreated or UVB irradiated $\left(15 \mathrm{~mJ} / \mathrm{cm}^{2}\right)$ were examined for the presence of AIF using the previously used monoclonal antibody. $\alpha$-tubulin
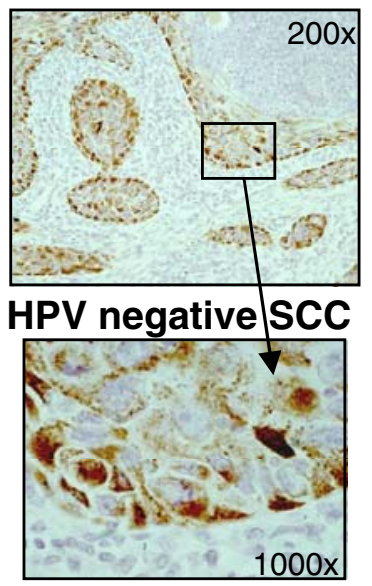
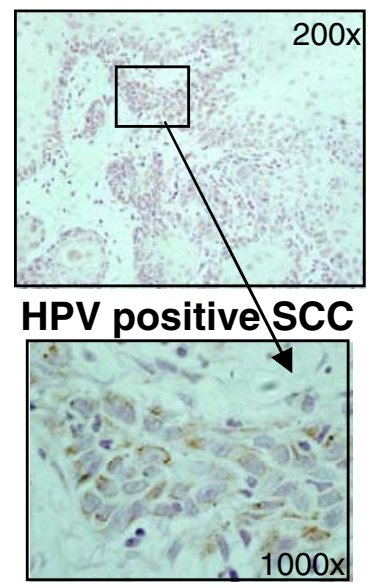

Fig. 4 AIF is confined to the cytoplasm in HPV positive tumors. Sections of HPV-typed human squamous cell carcinomas (SCCs) were stained using AIF antibody and counterstained with Haematoxylin and eosin. Pictures were taken at a magnification of $200 \times$ (top) and $1000 \times$ (bottom) using conventional microscopy. Note that AIF nuclear localization is only observed in the HPV negative biopsy. These images are representative of a multiple sample study described in Table 1
UV $24 \mathrm{~h}$

\section{B}

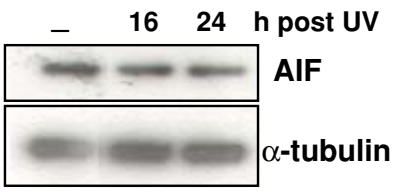

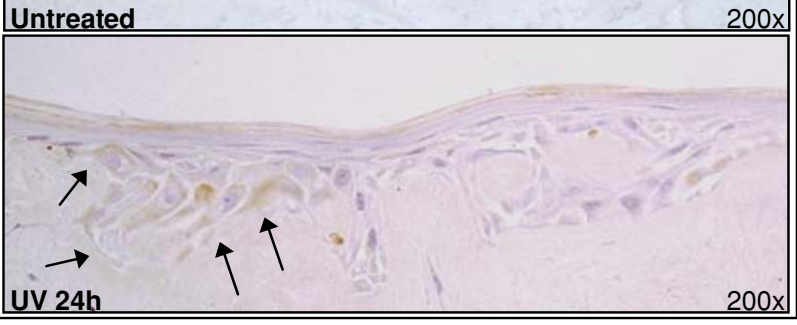

level was assayed as a control to normalize protein loading. (C) AIF translocation to the nucleus is inhibited in epithelium regenerated using human primary keratinocytes retroviraly expressing HPV8E6. Human skin sheets were reconstituted using keratinocytes transduced with empty control retrovirus vector (Mock) or with an HPV8E6-encoding retrovirus. AIF subcellular localization was determined in untreated or UVB irradiated $\left(45 \mathrm{~mJ} / \mathrm{cm}^{2}\right)$ cultures using AIF-selective antibody and DAB staining (stained brown). Pictures were taken by conventional microscopy. Bars represent $20 \mu \mathrm{m}$. The arrows point to the positive stained cells

negative tumors, $p=<0.0068$ according to Fisher's exact test (Table 1). This differential localization of AIF between HPV-positive and -negative tumors is in agreement with our earlier observations where AIF was confined to the cytoplasmic compartments of UV-irradiated regenerated epithelium (Fig. 3). These results suggest that AIF plays a part in a caspase-independent mechanism of apoptosis in the skin and that the inhibition of AIF nuclear translocation by HPV contributes to the lower apoptotic rates observed in HPV-positive compared to -negative lesions.

\section{Discussion}

Our previous studies have highlighted an important role for the anti-apoptotic activity of cutaneous HPVs in NMSC development, an activity that is encoded by the E6 protein that is capable of inhibiting simultaneously diverse UV-induced apoptotic pathways [20]. This results in an overall effect that facilitates the persistence of virally infected cells and alters the balance between proliferation and apoptosis in these can- 
Table 1 AIF nuclear localization is absent in HPV positive human tumors. Study of AIF immunostaining in a series of HPV-positive and -negative human squamous cell carcinomas stained as in Fig. 4. In HPV-negative (Neg) SCCs, AIF staining is strong and located in both the cytoplasm and nuclear compartments. In HPV-positive SCCs, AIF staining is weak ( $p$ value for AIF staining intensity between HPVpositive and -negative lesions $=0.0268$ according to the Chi squared test). Marked differences were apparent in the nuclear localization of AIF between the HPV-positive (0/8) versus the -negative tumors (6/9), $p=<0.0068$ according to Fisher's exact test

\begin{tabular}{llll}
\hline HPV typing & $\begin{array}{l}\% \text { age tumor } \\
+ \text { ve }\end{array}$ & $\begin{array}{l}\text { Staining } \\
\text { intensity }\end{array}$ & AIF localization \\
\hline Neg & $80 \%$ & ++ & cyto + nuclear \\
Neg & $100 \%$ & +++ & cyto + nuclear \\
Neg & $100 \%$ & +++ & cyto + nuclear \\
Neg & $40 \%$ & + & cyto \\
Neg & $80 \%$ & ++ & cyto \\
Neg & $100 \%$ & +++ & cyto + nuclear \\
Neg & $80 \%$ & +++ & cyto + nuclear \\
Neg & $80 \%$ & ++ & cyto \\
Neg & $95 \%$ & +++ & cyto + nuclear \\
$19-21$ & $10 \%$ & $+/-$ & cyto \\
$10-24-24$ & $80 \%$ & ++ & cyto \\
$14 D-37$ & $-v e$ & & \\
$14 D-37$ & $5 \%$ & $+/-$ & cyto \\
1 A-16 & $95 \%$ & ++ & cyto \\
15 rel & $10 \%$ & $-/+$ & cyto \\
16 & $-v e$ & & \\
Z95970 & $30 \%$ & ++ & cyto \\
\hline
\end{tabular}

cers [25]. In this study, we have investigated the molecular basis of E6 inhibition of apoptosis involving mitochondria.

Our results demonstrate that E6 maintains mitochondrial morphology and integrity to inhibit the release of proapoptotic factors including AIF, cytochrome $c$ and Omi. The mechanisms by which these and other factors are released from the mitochondria during apoptosis have been the focus of much recent research and still remain elusive [4]. While some studies have clearly demonstrated that caspases can trigger the permeabilization of the outer mitochondrial membrane that permits cytochrome $c$ and/or AIF release, other studies have indicated that AIF release from mitochondria was caspase-independent. Key proteins that control mitochondrial outer membrane permeabilization are Bax and Bak [5]. Multimerization of Bax and Bak, in association with other proteins such as tBid and lipid components, into pores has been proposed as a likely mechanism permitting release of IMS proteins, however there are marked differences between the proposed models in their ability to translocate different sized molecules [7, 38]. For example, reconstitution experiments in vitro have suggested a molecular sieve theory where Bax forms channels capable of allowing passage of small molecules such as cytochrome $c(16.6 \mathrm{kDa})$, but not the larger AIF (57 kDa) [14]. An alternate hypothesis invokes the formation of large pores capable of translocating molecules up to $2000 \mathrm{kDa}$ [15]. Our results using UVB as a physiologically important apoptotic inducer demonstrate for the first time that Bak is an essential component that is required for the release not only of cytochrome $c$, but also of larger molecules such as AIF from the mitochondria. Thus during UV-induced cell death in skin, Bak forms a nodal point in both the caspase dependent and -independent apoptotic pathways, that operates as the final checkpoint before irreversibility of the apoptotic signal and this provides a molecular explanation for the targeting of Bak for proteolysis by HPV E6 proteins. Silencing of Bak expression using shRNA generated a similar phenotype to cells expressing the HPV E6 protein, including the lack of mitochondrial fragmentation and loss of MOMP following UV. This suggests that the E6-mediated proteolysis of Bak is an essential viral activity that is necessary to prevent release of proapoptotic mitochondrial factors, thereby permitting the survival of virally-infected cells by abrogating simultaneously critical caspase-dependent and-independent pathways.

In addition to the inhibition of release of Omi and cytochrome $c$ by HPV5 E6, the retention of other survival factors in the mitochondria may also contribute towards cell survival. Survivin is a member of the inhibitor of apoptosis (IAP) family of proteins and has recently been reported to be present in mitochondria where it promotes cell survival and tumorigenesis [39]. Inhibition of Survivin activity using a dominant negative mutant lead to the rapid release of AIF from mitochondria and caspase-independent cell death in melanoma cells [40]. Whether this release was dependent upon Bak remains to be evaluated. The E6 protein of the oncogenic cervical HPV 16 was reported to increase Survivin expression, and that this was dependent upon the ability of the E6 protein to promote p53 proteolysis [41], although no increase in Survivin expression was seen in HeLa cells that express HPV18 E6, an E6 type that is also capable of promoting p53 degradation, as compared to non-HPV-containing cells [39]. However, as the E6 protein of cutaneous HPVs cannot promote $\mathrm{p} 53$ degradation is seems unlikely that this mechanism contributes towards cell survival, although this awaits further investigation. Since cutaneous HPV E6 proteins are able to signal Bak proteolysis [20,28], it is possible that the retention of Survivin in the mitochondria may also contribute towards cell survival.

In human skin, analysis of clonal patches of p53 cell patches has demonstrated that they have little or no precancerous potential indicating that other apoptotic mechanisms are likely to operate in the skin to eliminate damaged and potentially cancerous cells $[42,43]$. While it has been reported that DNA-damage-induced AIF translocation in neuronal cell death depends on p53 activity [44], and that p53 translocates to the mitochondria and interacts with Bak following DNA damage [45], our results using HPV18E6 demonstrate that AIF translocation and release of other IMS factors in 
response to UVB can function independently of p53 activity. The Bak-dependent release of AIF and other pro-apoptotic IMS factors provides an alternative pathway that can operate in cells carrying p53 mutations. To place our observations in a more physiologically relevant context, we showed that AIF translocation following UV damage also occurs in skin, demonstrating that caspase-independent pathways also play a part in the apoptotic response in this tissue, and that AIF translocation is inhibited by expression of HPV E6, most likely as a result of Bak proteolysis. The failure to initiate an apoptotic cascade, either caspase dependent or-independent, in HPV-infected cells following UV damage suggests that deleterious mutational changes may accumulate that further increase genetic instability in HPV-infected lesions.

Any perturbation of the apoptotic responses of cells by HPV-encoded proteins has important clinical implications for NMSC development. We therefore undertook an investigation to determine the sub-cellular localization of AIF in HPV-typed biopsy material. Immunostaining of tumors demonstrated that in HPV-negative lesions AIF nuclear translocation occurred. In contrast, in HPV-positive lesions AIF protein was restricted to the cytoplasmic compartment and no nuclear localization was ever observed. This result indicates that AIF plays a potentially important role in apoptosis in skin and that in HPV-associated NMSC AIF exclusion from the nucleus most likely contributes towards altering the balance between proliferation and apoptosis.

In summary, we have shown that AIF functions in an apoptotic pathway that removes UV-damaged cells from the epidermis and contributes towards apoptosis in NMSC. AIF release from the mitochondria following UV damage was dependent on Bak expression and was inhibited in HPV E6-expressing cells and HPV-positive NMSC. The restoration of Bak activity to HPV-infected cells may therefore provide a mechanism for the re-activation of both caspasedependent and -independent apoptotic mechanisms in intervention strategies in HPV-associated NMSC.

\section{Materials and methods}

\section{Cell culture and transfection}

HT1080 (human fibrosarcoma) cells were maintained in Dulbecco's modified Eagle's medium (DMEM) supplemented with $10 \%$ fetal calf serum and glutamine. HT1080 polyclonal cell lines expressing HPV 5 or 18 E6 were made as described previously [28]. Plasmid encoding Bak shRNA constructs or the empty vector (Mock) were transfected into HT1080 cells using a standard calcium phosphate method and monoclonal stable cell lines were then established. Tissue specimens used for immunohistochemistry were frozen in Cryobed mountant and stored at $-70^{\circ} \mathrm{C}$. Organotypic cul- tures were essentially prepared on de-epidermalized dermis as previously described [46]. Briefly, the reticular dermal surface was repopulated with human fibroblasts and keratinocytes were seeded on the papillary dermal surface and allowed to form a confluent monolayer. The culture was then raised to the air-liquid interface for a further 14 days before harvesting. For retroviral infection, the keratinocyte culture was infected with pLXSN or pLXSN-8E6. Where required, cells were irradiated with UVB using a UVP CL-1000 ultraviolet cross-linker with F8T5 bulbs giving a spectral peak at $312 \mathrm{~nm}$.

\section{Plasmid construction}

Three different shRNA templates directed against human Bak sequence (termed BI, BII and BIII) were designed and cloned into a shRNA expression vector-EGFP (generous gift from F.A. Rassendren). The 19-nt target sequences are for BI, 5'-CCGACGCTATGACTCAGAG$3^{\prime}$; for BII, 5'-GATTGCCACCAGCCTGTTT-3' and for BIII, 5'-TGGTCCCATCCTGAACGTG-3'. To generate the pLXSN-8E6 recombinant retroviral construct (kindly provided by Prof $\mathrm{H}$. Pfister), the HPV8 E6 ORF was amplified by PCR by using the following primers Fwd: GAAGCTTGAATTCTCTGACTTGTGCAATTTTCC; Bwd: AAGCTTGGATCCACAAAATCTTGCACAGTGACC, and cloned into BamH I/EcoR I digested pLXSN.

\section{Antibodies}

The monoclonal antibodies used were Bak (Ab-2, Calbiochem), p53 (D01, CRUK), AIF (E-1, Santa Cruz Biotechnology), Cytochrome $c$ (BD Biosciences), HtrA2/Omi (V-17, Santa Cruz Biotechnology), PCNA (PC10, CRUK), Cox4 (Molecular Probes), $\beta$-actin (Sigma) and $\alpha$-Tubulin (Calbiochem). The rabbit anti-mouse FITC conjugate and the Cy5 conjugated goat anti-mouse antibodies were obtained respectively from DAKO and Stratech.

\section{Western blot analysis}

HT1080 cells were washed once in ice cold PBS and lysed on ice for $30 \mathrm{~min}$ in lysis buffer (50 mM Tris pH 7.4, $150 \mathrm{mM}$ $\mathrm{NaCl}, 1 \%$ Nonidet-P40, $0.5 \%$ Sodium Deoxycholate, $0.1 \%$ SDS) supplemented with $1 \mathrm{x}$ cocktail protease inhibitors (Roche Diagnostics). The resulting extracts were sonicated and protein concentration was determined using the Bio-Rad Protein Assay (Bio-Rad). From keratinocyte cultures, proteins were extracted in $100 \mu$ l of lysis buffer, diluted in $2 \mathrm{x}$ Laemmli buffer and then heated at $100^{\circ} \mathrm{C}$ for $5 \mathrm{~min} .20 \mu \mathrm{g}$ of total protein or $10 \mu \mathrm{l}$ of each keratinocyte sample were separated on $12 \%$ SDS-PAGE and transferred onto PVDF 
membrane according to standard procedures. The membrane was probed with specific antibodies used at the following dilutions in 5\% powdered milk in PBST: anti-AIF 1:750, anti-p53 1:1000, anti-Bak 1:250 and anti- $\alpha$-tubulin 1:2000 (as a loading control). The secondary antibody was an HRPcoupled rabbit anti-mouse used at 1:1000 dilution (DAKO) and proteins were developed using the ECL \pm Plus kit (Amersham Pharmacia Biotech) according to manufacturers instructions. Cell fractionation was performed as described [48]. Cells were UV-irradiated with $15 \mathrm{~mJ} / \mathrm{cm}^{2}$ and harvested $24 \mathrm{~h}$ later.

Immunocytochemistry

Cells growing on coverslips were incubated for $30 \mathrm{~min}$ in $0.1 \mu$ M MitoTracker Orange CMTMRos (Molecular Probes) solution diluted in growth media at $37^{\circ} \mathrm{C}$ and then when required UV irradiated $\left(15 \mathrm{~mJ} / \mathrm{cm}^{2}\right)$. After $24 \mathrm{~h}$, cells were fixed in 4\% Paraformaldehyde and permeabilized for $1 \mathrm{~min}$ in PBS/0.1\% Triton-X100. Non-specific binding sites were blocked with horse serum for $5 \mathrm{~min}$, followed by incubation with the monoclonal anti-AIF antibody (1:100) for $90 \mathrm{~min}$ at room temperature. The secondary and tertiary antibodies were supplied in the ABC PK-6200 Universal kit (Vector Laboratories) and were used in accordance with the manufacturer's instructions. Nuclei were visualized by staining with $20 \mu \mathrm{g} / \mathrm{ml}$ Hoechst 33342 (Sigma). Images were captured on a LSM 510 inverted confocal microscope (Carl Zeiss MicroImaging, Inc.).

\section{Histological analyses}

Skin squamous cell carcinoma (SCC) was excised from immunocompetent patients. Each specimen was bisected, one half was fixed in $4 \%$ paraformaldehyde and processed for paraffin embedding, the other half was snapped frozen at the time of surgery and stored at $-70^{\circ} \mathrm{C}$. Tumor diagnosis was confirmed histologically. Ethical approval for this study was obtained from the East London and City Health Authority Research Ethics Committee and patient consent. HPV typing of biopsies was performed using a degenerate PCR strategy that we have previously demonstrated to detect all known HPV types [24]. For immunohistochemistry, $4 \mu \mathrm{m}$ thick paraffin embedded tissue sections were mounted and processed as previously described [47]. For antigen retrieval, sections were microwave treated under pressure for $4 \mathrm{~min}$ at $900 \mathrm{~W}$ in preheated citrate buffer $\mathrm{pH}$ 6.0. Sections were then blocked with horse serum to reduce non-specific binding and were subsequently incubated overnight with monoclonal anti-AIF antibody $(1: 100)$ at $4{ }^{\circ} \mathrm{C}$. Secondary biotinylated antibody (Vectastain Universal Elite ABC Kit Vector Laboratories, Peterborough, U.K) was then applied for $30 \mathrm{~min}$ at room temperature, followed by avidin biotin peroxidase complex for $20 \mathrm{~min}$. Sections were developed using diaminbenzidine (DAB), lightly counterstained using Gill's haematoxylin dehydrated in graded alcohols, then cleared in xylene and mounted using DePex (MetK Ltd.). No background staining was observed with anti-AIF or secondary antibody alone.

\section{Flow cytometry}

Cells were incubated for $30 \mathrm{~min}$ in media containing $0.1 \mu \mathrm{M}$ MitoTracker Orange CMTMRos (Molecular Probes) at $37^{\circ} \mathrm{C}$ and then untreated or UVB-irradiated at $15 \mathrm{~mJ} / \mathrm{cm}^{2}$. After $24 \mathrm{~h}$, cells were harvested, fixed in $0.25 \%$ Paraformaldehyde and permeabilized for $5 \mathrm{~min}$ in PBS/0.01\% Saponin (Sigma). Primary antibodies (AIF, Cytochrome $c$, Omi) were diluted 1:50 in PBS/0.01\% Saponin and incubated for 30 min at $4{ }^{\circ} \mathrm{C}$ with rotation. The secondary antibody, Cy5 Goat anti-mouse (1:100), was then incubated $30 \mathrm{~min}$ at $4^{\circ} \mathrm{C}$. Nuclei were stained with $20 \mu \mathrm{g} / \mathrm{ml}$ Hoechst 33342 (Sigma) for $3 \mathrm{~min}$. To quantify the release of mitochondrial proteins, labeled cells were analyzed by flow cytometry (LSR, Becton Dickinson) before and after homogenisation with a Dounce Homogenizer (Fisher) in Hepes buffer (20 mM Hepes-KOH pH7.4, $10 \mathrm{mM} \mathrm{KCl}, 1.5 \mathrm{mM} \mathrm{MgCl} 2,1 \mathrm{mM}$ EGTA, $1 \mathrm{mM}$ EDTA). To assay apoptosis, cells were stained with Annexin-V Alexa Fluor ${ }^{\circledR} 647$ conjugate (Cambridge BioScience) and DAPI (Sigma) and their profile were analyzed by flow cytometry (LSR). GFP-positive cells were gated and Annexin- $\mathrm{V}^{+}$, DAPI $^{-}$cells were scored as apoptotic. The loss of mitochondrial membrane potential $(\Delta \Psi m)$ was detected using the probe TMRE (tetramethylrhodamine ethyl ester, Molecular Probes). TMRE negative cells were scored as cells with depolarized mitochondrial membrane.

\section{Electron microscopy}

Cells were rinsed briefly with pre-warmed serum-free medium, then fixed with $4 \%$ paraformaldehyde and $2.5 \%$ glutaraldehyde in $0.1 \mathrm{M}$ Sorensens phosphate buffer for $1 \mathrm{~h}$ and then washed in same buffer before post fixation in potassium ferricyanide reduced $1 \%$ Osmium tetroxide for $1 \mathrm{~h}$. Cells were then washed in distilled water before being dehydrated in graded series of methanol and embedded in araldite resin. After polymerisation of the resin, ultrathin sections (90 nm) were cut on a Leica Ultracut S ultratome. The sections were contrasted with Uranyl acetate and lead citrate and viewed with a JEOL 1200 transmission electron microscope.

Acknowledgments The authors are grateful to Drs C.A. Harwood and C.M. Proby (University of London, UK) for tissue biopsies, and to Dr. S. Basu (Cancer Research UK, UK) for critical reading of the manuscript. We also thank Prof. H. Pfister (University of Cologne, Germany) for providing us the retroviral construct pLXSN-8-E6, and Dr. F.A. Rassendren (CNRS, France) for shRNA expression plasmids 
and helpful advice. This work was supported by funding from Cancer Research UK. B.A. was the recipient of a grant from the Dr. Mildred Scheel Stiftung / Deutsche Krebshilfe.

\section{References}

1. Danial NN, Korsmeyer SJ (2004) Cell death: critical control points. Cell 116(2):205-219

2. Kuwana T, Newmeyer DD (2003) Bcl-2-family proteins and the role of mitochondria in apoptosis. Curr Opin Cell Biol 15(6):691699

3. Mikhailov V et al (2003) Association of Bax and Bak homooligomers in mitochondria. Bax requirement for Bak reorganization and cytochrome c release. J Biol Chem 278(7):5367-5376

4. Green DR (2005) Apoptotic pathways: ten minutes to dead. Cell 121(5):671-674

5. Wei MC et al (2001) Proapoptotic BAX and BAK: a requisite gateway to mitochondrial dysfunction and death. Science 292(5517):727-730

6. Kandasamy K et al (2003) Involvement of proapoptotic molecules Bax and Bak in tumor necrosis factor-related apoptosis-inducing ligand (TRAIL)-induced mitochondrial disruption and apoptosis: differential regulation of cytochrome c and Smac/DIABLO release. Cancer Res 63(7):1712-1721

7. Green DR, Kroemer G (2004) The pathophysiology of mitochondrial cell death. Science 305(5684):626-629

8. Saelens $X$ et al (2004) Toxic proteins released from mitochondria in cell death. Oncogene 23(16):2861-2874

9. Susin SA et al (1999) Molecular characterization of mitochondrial apoptosis-inducing factor. Nature 397(6718):441-446

10. Cande $\mathrm{C}$ et al (2002) Apoptosis-inducing factor (AIF): key to the conserved caspase-independent pathways of cell death? J Cell Sci 115(Pt 24):4727-4734

11. Cregan SP, Dawson VL, Slack RS (2004) Role of AIF in caspase-dependent and caspase-independent cell death. Oncogene 23(16):2785-2796

12. Carter BZ et al (2003) Caspase-independent cell death in AML: caspase inhibition in vitro with pan-caspase inhibitors or in vivo by XIAP or Survivin does not affect cell survival or prognosis. Blood 102(12):4179-4186

13. Cande C et al (2004) AIF and cyclophilin A cooperate in apoptosis-associated chromatinolysis. Oncogene 23(8):1514 1521

14. Saito M, Korsmeyer SJ, Schlesinger PH (2000) BAX-dependent transport of cytochrome $\mathrm{c}$ reconstituted in pure liposomes. Nat Cell Biol 2(8):553-555

15. Kuwana T et al (2002) Bid, Bax, and lipids cooperate to form supramolecular openings in the outer mitochondrial membrane. Cell 111(3):331-342

16. Jemal A et al (2004) Cancer statistics 2004. CA Cancer J Clin 54(1):8-29

17. Goodwin RG, Holme SA, Roberts DL (2004) Variations in registration of skin cancer in the United Kingdom. Clin Exp Dermatol 29(3):328-330

18. Schwarz A et al (1995) Ultraviolet-B-induced apoptosis of keratinocytes: evidence for partial involvement of tumor necrosis factor-alpha in the formation of sunburn cells. J Invest Dermatol 104(6):922-927

19. Ichihashi $M$ et al (2003) UV-induced skin damage. Toxicology 189(1-2):21-39

20. Jackson S et al (2000) Role of Bak in UV-induced apoptosis in skin cancer and abrogation by HPV E6 proteins. Genes Dev 14(23):3065-3073
21. Shamanin V et al (1996) Human papillomavirus infections in nonmelanoma skin cancers from renal transplant recipients and nonimmunosuppressed patients. J Natl Cancer Inst 88(12):802-811

22. Harwood CA, Proby CM (2002) Human papillomaviruses and nonmelanoma skin cancer. Curr Opin Infect Dis 15(2):101-114

23. Pfister $\mathrm{H}$ (2003) Chapter 8: Human papillomavirus and skin cancer. J Natl Cancer Inst Monogr (31):52-56

24. Harwood CA et al (2000) Human papillomavirus infection and non-melanoma skin cancer in immunosuppressed and immunocompetent individuals. J Med Virol 61(3):289-297

25. Jackson $\mathrm{S}$ et al (2002) Reduced apoptotic levels in squamous but not basal cell carcinomas correlates with detection of cutaneous human papillomavirus. Br J Cancer 87(3):319-323

26. Storey A (2002) Papillomaviruses: death-defying acts in skin cancer. Trends Mol Med 8(9):417-421

27. Akgul B, Cooke JC, Storey A (2006) HPV-associated skin disease. J Pathology 208:165-175

28. Jackson S, Storey A (2000) E6 proteins from diverse cutaneous HPV types inhibit apoptosis in response to UV damage. Oncogene 19(4):592-598

29. de Villiers EM et al (2004) Classification of papillomaviruses. Virology 324(1):17-27

30. Nechushtan A et al (2001) Bax and Bak coalesce into novel mitochondria-associated clusters during apoptosis. J Cell Biol 153(6): 1265-1276

31. Arnoult D et al (2004) Cytomegalovirus cell death suppressor vMIA blocks Bax- but not Bak-mediated apoptosis by binding and sequestering Bax at mitochondria. Proc Natl Acad Sci USA 101(21):7988-7993

32. Cheng EH et al (2003) VDAC2 inhibits BAK activation and mitochondrial apoptosis. Science 301(5632):513-517

33. Qin JZ et al (2002) Regulation of apoptosis by p 53 in UV-irradiated human epidermis, psoriatic plaques and senescent keratinocytes. Oncogene 21(19):2991-3002

34. Joseph B et al (2002) Mitochondrial dysfunction is an essential step for killing of non-small cell lung carcinomas resistant to conventional treatment. Oncogene 21(1):65-77

35. Alonso M et al (2003) Flavopiridol induces apoptosis in glioma cell lines independent of retinoblastoma and p53 tumor suppressor pathway alterations by a caspase-independent pathway. Mol Cancer Ther 2(2):139-150

36. Gallego MA et al (2004) Apoptosis-inducing factor determines the chemoresistance of non-small-cell lung carcinomas. Oncogene 23(37):6282-6291

37. Green DR, Evan GI (2002) A matter of life and death. Cancer Cell 1(1):19-30

38. Sharpe JC, Arnoult D, Youle RJ (2004) Control of mitochondrial permeability by Bcl-2 family members. Biochim Biophys Acta 1644(2-3):107-113

39. Dohi $\mathrm{T}$ et al (2004) Mitochondrial survivin inhibits apoptosis and promotes tumorigenesis. J Clin Invest 114(8):11171127

40. Liu T, Brouha B, Grossman D (2004) Rapid induction of mitochondrial events and caspase-independent apoptosis in Survivintargeted melanoma cells. Oncogene 23:39-48

41. Borbely A et al (2006) Effects of human papillomavirus type 16 oncoproteins on survivin gene expression. J Gen Virol 87:287294

42. Jonason AS et al (1996) Frequent clones of p53-mutated keratinocytes in normal human skin. Proc Natl Acad Sci USA 93(24):14025-14029

43. Ren ZP et al (1996) Human epidermal cancer and accompanying precursors have identical p53 mutations different from p53 mutations in adjacent areas of clonally expanded non-neoplastic keratinocytes. Oncogene 12(4):765-773 
44. Cregan SP et al (2002) Apoptosis-inducing factor is involved in the regulation of caspase-independent neuronal cell death. J Cell Biol 158(3):507-517

45. Leu JI et al (2004) Mitochondrial p53 activates Bak and causes disruption of a Bak-Mcl1 complex. Nat Cell Biol 6(5):443450

46. Akgul B et al (2005) The E7 protein of cutaneous human papillomavirus type 8 causes invasion of human keratinocytes into the dermis in organotypic cultures of skin. Cancer Res 65(6):22162223

47. Bull JJ et al (2001) Contrasting localization of c-Myc with other Myc superfamily transcription factors in the human hair follicle and during the hair growth cycle. J Invest Dermatol 116(4):617622

48. Mihara M, Moll UM (2003) Detection of mitochondrial localization of p53. Methods Mol Biol 234:203-209 